To appear in Proc. 2011 ACC

\title{
Synchronization on the N-Torus with Noisy Measurements
}

\author{
Nina Mahmoudian and Derek A. Paley
}

\begin{abstract}
We consider the problem of cooperative control of unmanned vehicles in an uncertain environment in which each vehicle can obtain only noisy measurements of the other vehicles. In particular, we want to establish convergence to a desired formation using a stochastic algorithm with measurement errors. In this paper, the problem of stabilization of parallel formations in a self-propelled particle model is considered, which can be modeled as synchronization on the $N$-torus in the presence of noisy measurements of relative phase. Simulations are included to illustrate the result.
\end{abstract}

\section{INTRODUCTION}

The increasing interest in applications of multi-agent autonomous systems such as unmanned air vehicles, mobile robots, and unmanned underwater vehicles increases the need for cooperative-control algorithms to address real-world challenges. A networked multi-vehicle system may have only limited knowledge of its environment and the states of the other agents. One such limitation is the issue of noisy communication links. With imperfect communication, convergence of a standard consensus algorithm is not guaranteed.

A consensus algorithm specifies the interaction between each agent and its neighbors; consensus occurs when every agent reaches a common value. There is a rich body of literature on consensus protocols in Euclidean space in both continuous time and discrete time, with applications to multiagent autonomous systems [6], [7] (see [8], [9] for a review). In addition, the problem of coordination and consensus with noisy communication has been addressed using various approaches. The majorization of a stress function is used in [3] to develop a distributed coordination algorithm that stabilizes the shape of a relative sensing network to a desired formation; robustness of the algorithm against measurement errors in the relative information of neighboring agents is established. Robustness of consensus to noise for directed communication characterized by the $H_{2}$ norm of the system is studied in [15]. A stochastic adaptive algorithm approach is used in [4], [5], in which a stochastic approximation type algorithm with a decreasing step size is proposed and convergence results are established with measurement noise.

There have been other works that consider noise-free consensus on non-Euclidean manifolds such as the $N$-torus [11],

This material is based upon work supported by the National Science Foundation under Grant Nos. CMMI0928416 and CMMI0954361 and the Office of Naval Research under Grant No. N00014-09-1-1058.

N. Mahmoudian is an assistant professor in the Department of Mechanical Engineering-Engineering Mechanics, Michigan University of Technology, Houghton, MI 49931, USA ninamemtu.edu

D. Paley is an assistant professor in the Department of Aerospace Engineering, University of Maryland, College Park, MD 20742, USA dpaley@umd.edu
[12] and the sphere [10]. The problem of synchronization and global convergence on the $N$-torus has been studied using noise-free state iteration by assuming exact data exchange between the agents [11], [12]. Each agent updates its heading based on the relative phases of its neighbors on the torus.

The contribution of this paper is to present convergence analysis in consensus seeking on the $\mathrm{N}$-torus in an uncertain environment where each agent can only obtain noisy measurements of its neighbors' relative phases. We study the behavior of a discrete-time network of $N$ agents moving on the torus. To deal with the measurement noise, a stochastic approximation algorithm is proposed for consensus seeking where the signal received from other agents is corrupted by additive noise. Convergence to the set of synchronized phases is established for all-to-all interactions by constructing a stochastic Lyapunov function. We assume the corrupting noise is associated with signal reception, not transmission, and that it has zero mean in the range $[-\pi / 2, \pi / 2]$.

The paper is organized as follows. Section II reviews the problem of synchronization on the $N$-torus and consensus. It also summarizes existing convergence results for Euclidean consensus in the presence of noisy measurement using stochastic Lyapunov analysis. Section III presents a stochastic Lyapunov function for synchronization on the $N$-torus following the first-order Euler approximation of the continuous-time algorithm, establishes the conditions for convergence, and presents simulation results. A stochastic Lyapunov function for synchronization on the $N$-torus for discrete-time is introduced in Section IV. Section V summarizes the paper and indicates ongoing research directions.

\section{BACKGROUND AND MATHEMATICAL PRELIMINARIES}

In this section, the problems of synchronization on the $N$-torus and Euclidean consensus are reviewed. In addition, existing convergence results for Euclidean consensus in the presence of noisy measurement are summarized.

Consider a network of $N$ identical agents interacting via an undirected graph $G=(\mathcal{N}, \mathcal{E})$ with nodes $\mathcal{N}=$ $\{1,2, \ldots, N\}$ and edges $\{l, k\} \in \mathcal{E} \subset \mathcal{N} \times \mathcal{N}$. The set of neighbors of node $k$ is represented by $\mathcal{N}_{k}=\{l \in$ $\mathcal{N} \mid(l, k) \in \mathcal{E}\}$. The graph $G$ is called undirected when $(l, k) \in \mathcal{N}_{k} \Leftrightarrow(k, l) \in \mathcal{N}_{k}$. The graph $G$ corresponding to all-to-all communication satisfies $(k, l) \in \mathcal{E} \forall l \neq k$.

The Laplacian $\mathbf{L}$ associated to the graph $G$ is defined as the following $N \times N$ matrix:

$$
\mathbf{L}_{k l}= \begin{cases}d_{k}, & \text { if } l=k, \\ -1, & \text { if } l \in \mathcal{N}_{k}, \\ 0, & \text { otherwise, }\end{cases}
$$


where $d_{k}=\left|\mathcal{N}_{k}\right|$ is the number of neighbors of agent $k$. If graph $G$ is undirected then $\mathbf{L}$ is symmetric positive semidefinite. If $G$ is also connected then $\operatorname{rank}(\mathbf{L})=N-1$ and the null space of $\mathbf{L}$ is spanned by $1_{N}=[1, \ldots, 1]^{T} \in \mathbb{R}^{N}$.

\section{A. Synchronization on the $N$-Torus}

Continuous-time algorithm: Consider a network of $N$ identical agents evolving on the unit circle $S^{1}$. ( For example, the synchronization of vehicle headings in the plane evolves on the $S^{1}$.) The state of each agent at time $t$ can be represented by $\theta^{k} \in S^{1}, k \in \mathcal{N}$. The state space is the torus, $T^{N} \triangleq S^{1} \times \ldots \times S^{1}$ and the agents communicate pairwise. The goal is to stabilize the set of synchronized formations in which $\theta^{k}=\theta^{l} \forall k, l$. In the synchronized set the centroid of the phasors

$$
p_{\theta} \triangleq \frac{1}{N} \sum_{k \in \mathcal{N}} e^{i \theta^{k}} \triangleq \frac{1}{N} \sum_{k \in \mathcal{N}} z^{k}
$$

satisfies $\left|p_{\theta}\right|=1$.

Consider the all-to-all potential [13]

$$
U(\theta)=\frac{N}{2}\left|p_{\theta}\right|^{2}
$$

which can be written in terms of the Laplacian phase potential, $P=Q_{L}=\frac{1}{2 N}\left\langle e^{i \theta}, \mathbf{L} e^{i \theta}\right\rangle$, where $e^{i \theta}=$ $\left[e^{i \theta^{1}}, \ldots, e^{i \theta^{N}}\right]^{T}$. We have

$$
U(\theta)=\frac{N}{2}-\frac{1}{2 N}\left\langle e^{i \theta}, \mathbf{L} e^{i \theta}\right\rangle=\frac{N}{2}-P,
$$

where $\langle.,$.$\rangle is the inner product. The gradient of U(\theta)$ is

$$
\frac{\partial U}{\partial \theta^{k}}=-\frac{1}{N}\left\langle i e^{i \theta^{l}}, \mathbf{L}_{k} e^{i \theta}\right\rangle=-\frac{1}{N} \sum_{k \in \mathcal{N}} \sin \left(\theta^{l}-\theta^{k}\right),
$$

where $\mathbf{L}_{k}$ is the $k$ th row of the the $\mathbf{L}$.

A continuous-time gradient algorithm associated with the potential is [13]

$$
\dot{\theta}^{k}=K \frac{\partial U}{\partial \theta^{k}}=-\frac{K}{N} \sum_{k \in \mathcal{N}} \sin \left(\theta^{l}-\theta^{k}\right) .
$$

This control requires measurements of the relative phases $\theta^{l}-\theta^{k}$. The potential $U(\theta)$ reaches its unique maximum when all phases are identical (synchronization). When $K<$ 0 , the set of synchronized states is asymptotically stable and all other critical points of $U(\theta)$ are unstable [13].

When $K<0$, the same result equivalently holds in a rotating frame, that is, for the coupled-phase oscillators model [13]

$$
\dot{\theta}^{k}=\omega_{0}-\frac{K}{N} \sum_{k \in \mathcal{N}} \sin \left(\theta^{l}-\theta^{k}\right) .
$$

In (5) all oscillators have identical natural frequency $\omega_{0}$ and each oscillator is modeled by a phase variable $\theta^{k}$. This model is a simplified version of the Kuramoto model, where in general the oscillators have different natural frequencies. Synchronization of the Kuramoto model have been studied [1], [2], including oscillators with noisy, randomly distributed intrinsic frequencies and time-delayed interactions [14]. This paper addresses the problem of synchronization in the presence of noisy measurement of the relative phases $\theta^{l}-\theta^{k}$, as depicted in Figure 1 .

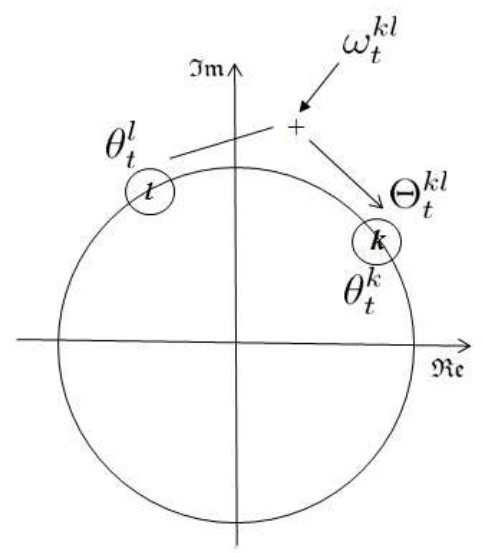

Fig. 1. Phase measurement with additive noise $\omega_{t}^{k l}$.

Discrete-time algorithm: Let $\theta_{t}^{k} \in S^{1}, k \in \mathcal{N}$, be the state of each agent at time $t$ or, equivalently, the unit phasor $z_{t}^{k} \in \mathbb{C},\left|z_{t}^{k}\right|=1$. Consider a network of $N$ identical agents evolving on the unit circle. The state equation of each agent $k$ in a discrete-time synchronization algorithm is [11], [12]

$$
\theta_{t+1}^{k}=\arg \left(\left(1-a^{k}\right) z_{t}^{k}+a^{k} \frac{1}{d_{k}} \sum_{l \in \mathcal{N}_{k}} z_{t}^{l}\right),
$$

where $a^{k} \in(0,1)$. For the case of a fixed, undirected graph, the algorithm (6) is a discrete-time approximation of a gradient algorithm for the Laplacian phase potential, $Q_{L}=\frac{1}{2 N}\left\langle e^{i \theta_{t}}, \mathbf{L} e^{i \theta_{t}}\right\rangle$, where $e^{i \theta_{t}}=\left[e^{i \theta_{t}^{1}}, \ldots, e^{i \theta_{t}^{N}}\right]^{T}$. The states are updated either asynchronously or synchronously with small $a^{k}$ [11]. A dynamic approach was proposed in [12], which uses the local information from the Euclidean consensus algorithm to estimate the global information required for synchronization on the $N$-torus:

$$
\begin{aligned}
& z_{t+1}^{k}=z_{t}^{k}-\mathbf{D}_{d} \mathbf{L}_{k} z_{t} \\
& \theta_{t+1}^{k}=\arg \left(\left(1-a^{k}\right) z_{t}^{k}+a^{k} \frac{1}{d_{k}} \sum_{l \in \mathcal{N}_{k}} z_{t}^{l}\right)
\end{aligned}
$$

where $\mathbf{D}_{d}=\operatorname{diag}\left(d_{1}^{-1}, \ldots, d_{N}^{-1}\right)$. The algorithm (7) is expressed in terms of the relative phasors $z_{t}^{l}-z_{t}^{k}$ for the convergence analysis.

If $G=G(t)$ is uniformly connected and $\mathbf{L}=\mathbf{L}(t)$ is bounded and piecewise continuous [12], then algorithm (7) asymptotically converges to a critical point of $U(\theta)[12$, theorem 3]. The only stable set of equilibrium points is the synchronized set characterized by $N$ identical phases.

\section{B. Consensus in the Presence of Noise}

Let $z_{t}^{k} \in \mathbb{R}$ be the state of agent $k$ at time $t \in \mathbb{Z}^{+}$. Denote the state vector $z_{t}=\left[z_{t}^{1}, \ldots, z_{t}^{N}\right]^{T}$. A discrete-time 
synchronous consensus algorithm for each agent is [8]

$$
z_{t+1}^{k}=z_{t}^{k}+\frac{a^{k}}{d_{k}} \sum_{l \in \mathcal{N}_{k}}\left(z_{t}^{l}-z_{t}^{k}\right)
$$

where $a^{k} \in(0,1)$ is the step size for agent $k$. Consensus is achieved by a network of agents if $\left|z_{t}^{k}-z_{t}^{l}\right| \rightarrow 0$ as $t \rightarrow \infty, \forall k \neq l$.

For the case that agent $k$ receives noisy measurements of the states of its neighbors, $q_{t}^{k l}$ is the resulting measurement of agent $l$ 's states by agent $k$ [4]:

$$
q_{t}^{k l}=z_{t}^{l}+w_{t}^{k l}, \quad t \in \mathbb{Z}^{+}, \quad l \in \mathcal{N}_{k},
$$

where additive noise $\left\{w_{t}^{k l}, t \in \mathbb{Z}^{+}, k \in \mathcal{N}, l \in \mathcal{N}_{k}\right\}$ is introduced as shown in Figure 2.

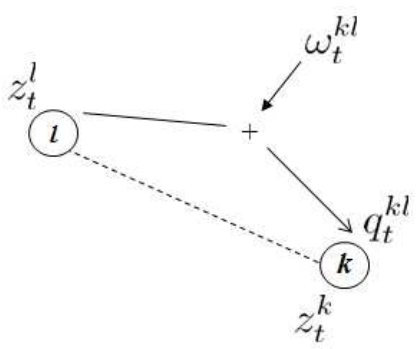

Fig. 2. State measurement with additive noise $w_{t}^{k l}$.

From [4], a stochastic consensus algorithm is (assuming $\left.a_{t}^{k}=a_{t} \in[0,1] \forall k, t\right)$

$$
z_{t+1}^{k}=\left(1-a_{t}\right) z_{t}^{k}+\frac{a_{t}}{d_{k}} \sum_{l \in \mathcal{N}_{k}} q_{t}^{k l}, t \in \mathbb{Z}^{+} .
$$

We rewrite (9) to adopt the structure of the recursion used in the classic stochastic approximation algorithm [4]

$$
z_{t+1}^{k}=z_{t}^{k}+\frac{a_{t}}{d_{k}} \sum_{l \in \mathcal{N}_{k}}\left(q_{t}^{k l}-z_{t}^{k}\right)
$$

Note (10) only requires (noisy) measurements of the relative states, $z_{t}^{l}-z_{t}^{k}+\omega_{t}^{k l}$.

The objective is to select a step size so that agents converge to a common limit in a certain sense. To characterize the asymptotic behavior of the agents, the following definitions are used [4]:

- Weak consensus: $E\left|z_{t}^{k}\right|^{2}<\infty \forall t \geq 0, k \in \mathcal{N}$, and

$$
\lim _{t \rightarrow \infty} E\left|z_{t}^{k}-z_{t}^{l}\right|^{2}=0 \forall k, l \in \mathcal{N},
$$

where $E$ is the expected value operator.

- Mean square consensus: $E\left|z_{t}^{k}\right|^{2}<\infty \forall t \geq 0, k \in \mathcal{N}$, and there exists a random variable $z^{*}$ such that

$$
\lim _{t \rightarrow \infty} E\left|z_{t}^{k}-z^{*}\right|^{2}=0 \forall k \in \mathcal{N} \text {. }
$$

- Strong consensus: There exists a random variable $z^{*}$ such that

$$
\lim _{t \rightarrow \infty} z_{t}^{k}=z^{*} \text { with probability one } \forall k \in \mathcal{N} .
$$

Consider the following conditions and assumptions for the additive noise and step size [4]:

(A1) The noises $w_{t}^{k l}$ are independent with respect to indices $k, l, t$ and the initial state vector $z_{0}$, where $E\left|z_{0}\right|^{2}<$ $\infty$. Each $w_{t}^{k l}$ has zero mean and variance $Q_{t}^{k l} \geq 0$, where $\sup _{t \geq 0, k \in \mathcal{N}} \sup _{l \in \mathcal{N}_{k}} Q_{t}^{k l}<\infty$.

(A2) The sequence $a_{t}, t \geq 0$, satisfies $a_{t} \in[0,1]$, $\sum_{t=0}^{\infty} a_{t}=\infty$, and $\sum_{t=0}^{\infty} a_{t}^{2}<\infty$.

(A3) The graph $G$ is undirected and connected.

[4, Theorem 21] shows that under assumptions (A1)(A3) the expected value of the difference between the states of any two agents converges to zero and, therefore, algorithm (9) achieves weak consensus.

A stochastic Lyapunov function can be defined based on the state differences of every pair of connected agents averaged over multiple random trials. Let potential $P_{t}^{k}$ for agent $k$ be [4]

$$
P_{t}^{k}=\frac{1}{2} \sum_{l \in N_{k}}\left|z_{t}^{k}-z_{t}^{l}\right|^{2}, \quad t \geq 0 .
$$

Accordingly, the total potential is $P_{t}=\sum_{k \in \mathcal{N}} P_{t}^{k}$ and the Lyapunov function $V_{t}$ is the expected value of the total potential,

$$
V_{t}=E\left(\sum_{k \in \mathcal{N}} P_{t}^{k}\right)=E\left(P_{t}\right), t \geq 0 .
$$

In terms of the graph Laplacian we have

$$
P_{t}=z_{t}^{T} \mathbf{L} z_{t} \text { and } V_{t}=E\left(z_{t}^{T} \mathbf{L} z_{t}\right)
$$

where $z_{t}=\left[z_{t}^{1}, \ldots, z_{t}^{N}\right]^{T}$.

[4, Theorem 26] states that, under assumptions (A1)(A3), algorithm (9) achieves mean square consensus. The result is based on asymptotic vanishing of $V_{t}$, which indicates that the state vector $z_{t}$ will approach the subspace spanned by $1_{N}$. This is proven by defining the directions of invariance associated with the consensus algorithm (9), which is characterized in terms of the degree of each agent in the network. The proof shows that the oscillation of the sequence $z_{t}, t \geq 0$ along the direction $1_{N}$ will gradually die off.

\section{NOISY SYNCHRONIZATION ON N-TORUS: CONTINUOUS-TIME Algorithm}

Synchronization on the $N$-torus requires knowledge of the relative orientations of at least some of the agents. In this section, we study the case that agent $k$ receives measurements of the relative orientation of every other agent, albeit corrupted by noise. The results extend naturally to connected, undirected graphs. We have a network of $N$ identical agents evolving on the torus, where each agent $k \in \mathcal{N}$ receives noisy measurements of the relative state of 
its neighbors (see Figure 1).

Consider the following two models for the noise $\left\{\omega_{t}^{k l}, t \in\right.$ $\left.\mathbb{Z}^{+}, k \in \mathcal{N}, l \in \mathcal{N}_{k}\right\}$ :

(N1) The additive noises are independent and identically distributed with respect to the indices $k, l, t$ (Figure 3).

(N2) The additive noise on each agent is the same for every measurement at time $t$ (Figure 4).

The first noise model is more general and is not addressed here; we adopt the second model, which we associate with noisy reception (as opposed to noisy transmission) of information.

We make the following additional assumptions:

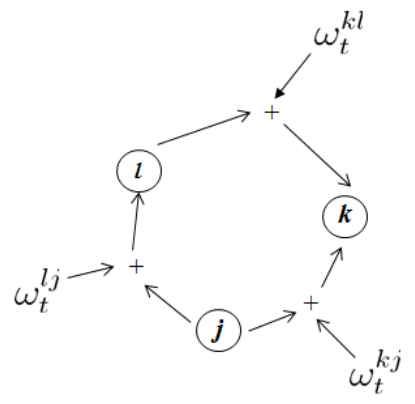

Fig. 3. Additive noise model (N1): $\omega_{t}^{k l} \neq \omega_{t}^{k j} \neq \omega_{t}^{l j}$.

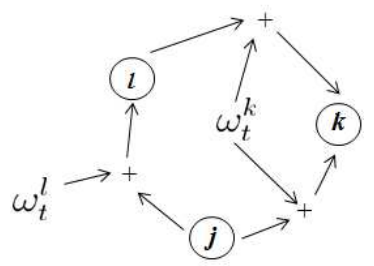

Fig. 4. Additive noise model (N2): $\omega_{t}^{k l}=\omega_{t}^{k j}=\omega_{t}^{k} \neq \omega_{t}^{l}$.

$\left(\mathrm{A} 1^{\prime}\right)$ The graph $G$ corresponds to an all-to-all communication network.

$\left(\mathrm{A} 2^{\prime}\right)$ The additive noise on agent $k$ obeys noise model (N2), i.e., $\omega_{t}^{k l}=\omega_{t}^{k j}=\omega_{t}^{k} \quad \forall l, j \in \mathcal{N}_{k}$, where $w_{t}^{k}$ has zero mean and finite range $\sup _{t \geq 0, k \in \mathcal{N}} \sup _{l \in \mathcal{N}_{k}} \omega_{t}^{k} \leq \pi / 2$.

Using a first-order Euler approximation, the continuoustime algorithm becomes

$$
\theta_{t+1}^{k}=\theta_{t}^{k}-\frac{a_{t} K}{N} \sum_{k \in \mathcal{N}} \sin \left(\theta_{t}^{l}-\theta_{t}^{k}\right),
$$

which depends on the relative phase $\theta_{t}^{l}-\theta_{t}^{k}$ measurement and $a_{t} \in[0,1]$. As shown in (2) the phase potential at each instant can be calculated from

$$
U_{t}=\frac{N}{2}-\frac{1}{2 N}\left\langle e^{i \theta_{t}}, \mathbf{L} e^{i \theta_{t}}\right\rangle=\frac{N}{2}-P_{t} .
$$

From (3), in discrete time we get

$$
U_{t+1}=U_{t}-\frac{a_{t} K}{N}\left\langle i e^{i \theta_{t}^{l}}, \mathbf{L}_{k} e^{i \theta_{t}}\right\rangle^{2} .
$$

Assume that the relative phase measurement $\theta_{t}^{l}-\theta_{t}^{k}$ by agent $k$ is corrupted by noise $\omega_{t}^{k}$ at each instant. The result of measurement of l's state by $k$ is $\theta_{t}^{l}+\omega_{t}^{k}$, hence the gradient (3) becomes

$$
\begin{aligned}
\frac{\partial U}{\partial \theta_{t}^{k}} & =-\frac{1}{N}\left\langle i e^{i\left(\theta_{t}^{l}+\omega_{t}^{k}\right)}, \mathbf{L}_{k} e^{i \theta_{t}}\right\rangle \\
& =-\frac{1}{N} \sum_{k \in \mathcal{N}} \sin \left(\theta_{t}^{l}-\theta_{t}^{k}+\omega_{t}^{k}\right) .
\end{aligned}
$$

According to $\left(\mathrm{A} 2^{\prime}\right)$, the noise lies in a compact distribution on the interval of $[-\pi / 2, \pi / 2]$ with zero mean. Let $\alpha_{t}^{k}=$ $\theta_{t}^{l}-\theta_{t}^{k}-\pi / 2$, which implies

$$
\theta_{t+1}^{k}=\theta_{t}^{k}-\frac{a_{t} K}{N} \sum_{k \in \mathcal{N}} \cos \left(\alpha_{t}^{k}+\omega_{t}^{k}\right) .
$$

The aim is to build a stochastic Lyapunov function based on the potential function $U_{t}$ and perform stability analysis of (14). By analogy with (13) the evolution of the phase potential becomes

$$
\begin{aligned}
U_{t+1} & =U_{t}-\frac{a_{t} K}{N}\left\langle i e^{i\left(\theta_{t}^{l}+\omega_{t}^{k}\right)}, \mathbf{L}_{k} e^{i \theta_{t}}\right\rangle\left\langle i e^{i \theta_{t}^{l}}, \mathbf{L}_{k} e^{i \theta_{t}}\right\rangle \\
& =U_{t}-\frac{a_{t} K}{N} \sum_{k \in \mathcal{N}}\left\langle i e^{i\left(\theta_{t}^{l}+\omega_{t}^{k}\right)}, e^{i \theta_{t}^{k}}\right\rangle\left\langle i e^{i \theta_{t}^{l}}, e^{i \theta_{t}^{k}}\right\rangle .
\end{aligned}
$$

Following [4], the mean of the total potential is the stochastic Lyapunov function $V_{t}=E\left(Q_{L_{t}}\right)=E\left(P_{t}\right)$. By (12), considering the definition of $\alpha_{t}^{k}$, we have,

$$
\begin{aligned}
P_{t+1}= & P_{t}+\frac{a_{t} K}{N} \sum_{k \in \mathcal{N}} \cos \alpha_{t}^{k} \cos \left(\alpha_{t}^{k}+\omega_{t}^{k}\right) \\
= & P_{t}+\frac{a_{t} K}{N} \sum_{k \in \mathcal{N}}\left(\cos ^{2} \alpha_{t}^{k} \cos \omega_{t}^{k}\right. \\
& \left.+\frac{1}{2} \sin 2 \alpha_{t}^{k} \sin \omega_{t}^{k}\right) .
\end{aligned}
$$

Hence, we take the expectation of both sides of (15).

By assumption $\left(\mathrm{A} 2^{\prime}\right), \cos \omega_{t}^{k} \geq 0$ for all $k$ and $t$, hence $\cos ^{2} \alpha_{t}^{k} \cos \omega_{t}^{k} \geq 0$. For the second term, $E\left(\sin \omega_{t}^{k}\right)=0$ and the independence of $\alpha_{t}^{k}$ and $\omega_{t}^{k}$ gives

$$
E\left(\sin 2 \alpha_{t}^{k} \sin \omega_{t}^{k}\right)=E\left(\sin 2 \alpha_{t}^{k}\right) E\left(\sin \omega_{t}^{k}\right)
$$

Therefore, expected value of the second term is zero. Consequently with $K<0$, the Lyapunov function is non-increasing; $V_{t+1} \leq V_{t}$, which implies $\lim _{t \rightarrow \infty} V_{t}=$ $\lim _{t \rightarrow \infty} E\left(P_{t}\right)=0$. Since $\left\langle e^{i \theta_{t}}, \mathbf{L} e^{i \theta_{t}}\right\rangle=\sum_{(l, k) \in \mathcal{E}} \mid e^{i \theta_{t}^{k}}-$ $\left.e^{i \theta_{t}^{l}}\right|^{2}$, it follows that

$$
\lim _{t \rightarrow \infty} E\left|e^{i \theta_{t}^{k}}-e^{i \theta_{t}^{l}}\right|^{2}=0 \forall k, l \in \mathcal{N} .
$$

Theorem: Under assumptions (A1')-(A2'), algorithm (14) achieves weak consensus on the $N$-torus.

To illustrate synchronization of a set of agents in the presence of noisy communication, we consider a graph Laplacian corresponding to all-to-all communication of five agents $(N=5)$. 
The initial conditions of the simulation are picked randomly from $[0,2 \pi]$. The measurement noise has a truncated normal distribution in the range $[-\pi / 2, \pi / 2]$. Figure 5 shows the weak convergence of algorithm (14) where the measurement noise has standard deviation is $\sigma=10$ and zero mean. Figure 6 illustrates the potential $P_{t}$ for ten trials and the resulting stochastic Lyapunov function, $V_{t}=E\left(P_{t}\right)$.

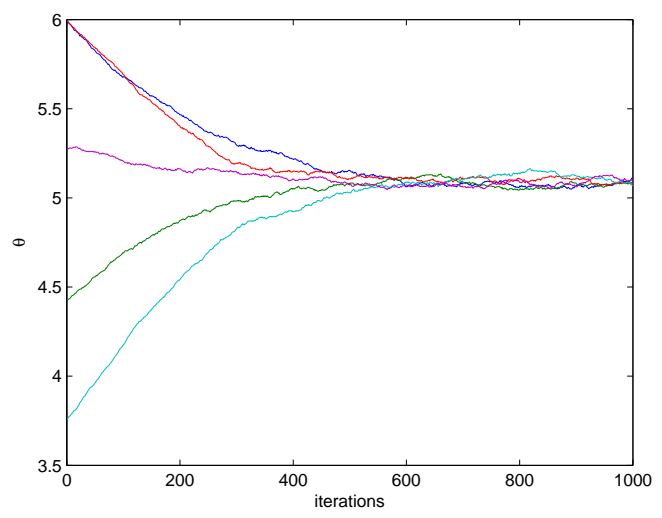

Fig. 5. Five agents reach weak consensus on the torus with noise standard deviation $\sigma=10$.

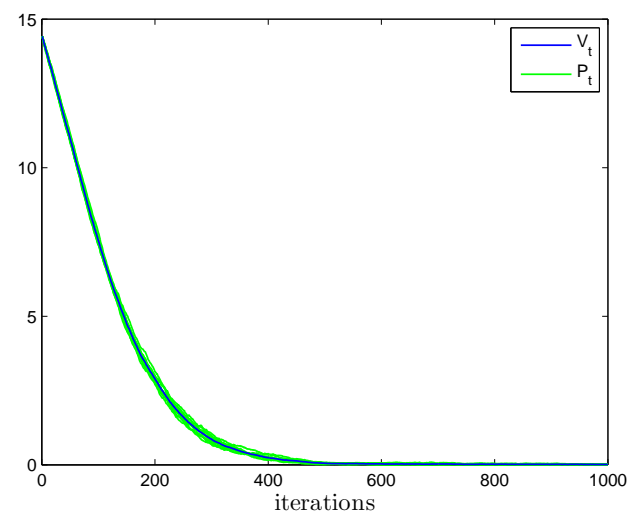

Fig. 6. The potential of five agents for ten trials with $\sigma=10$ and the stochastic Lyapunov function.

\section{NOISY SYNCHRONIZATION ON N-TORUS: DisCRETE-TIME AlgorithM}

Let $\Theta_{t}^{k l}$ be the result of the measurement of l's state by $k$ :

$$
\Theta_{t}^{k l}=\theta_{t}^{l}+\omega_{t}^{k}, \quad t \in \mathbb{Z}^{+}, \quad l \in \mathcal{N}_{k},
$$

where $w_{t}^{k} \in \mathbb{R}$ is additive noise satisfying (A2'). Let $z_{t}^{l}=$ $e^{i \theta_{t}^{l}}$ and $\Omega_{t}^{k l}=e^{i \omega_{t}^{k}}$, so that $z_{t}^{k l} \triangleq e^{i \Theta_{t}^{k l}}=z_{t}^{l} \Omega_{t}^{k l}$.

Augmenting algorithm (6) with the noise model yields the following update rule:

$$
\theta_{t+1}^{k}=\arg \left(\left(1-a_{t}\right) z_{t}^{k}+a_{t} \frac{1}{d_{k}} \sum_{l \in \mathcal{N}_{k}} z_{t}^{l} \Omega_{t}^{k l}\right) .
$$

To conduct the stability analysis of (16) following [4], the total potential can be defined in terms of the graph Laplacian

$$
P_{t}=z_{t}^{T} \mathbf{L} z_{t}
$$

and the mean of the total potential is the stochastic Lyapunov function

$$
V_{t}=E\left(P_{t}\right)
$$

Consider the following additional assumption:

$\left(\mathrm{A} 3^{\prime}\right)$ The sequence $a_{t}, t \geq 0$, satisfies $a_{t} \in(0,1)$, $\sum_{t=0}^{\infty} a_{t}=\infty$, and $\sum_{t=0}^{\infty} a_{t}^{2}<\infty$.

From equation (16), we have

$$
\begin{aligned}
\theta_{t+1}^{k} & =\arg \left[a_{t} \frac{1}{d_{k}} \sum_{l \in \mathcal{N}_{k}} z_{t}^{l} \Omega_{t}^{k}+\left(1-a_{t}\right) z_{t}^{k}\right] \\
& =\arg \left[a_{t} \frac{1}{d_{k}} \sum_{l \in \mathcal{N}_{k}}\left(z_{t}^{l} \Omega_{t}^{k}-z_{t}^{k} \Omega_{t}^{k}\right)+\left(1-a_{t}+a_{t} \Omega_{t}^{k}\right) z_{t}^{k}\right] \\
& =\arg \left[a_{t} \frac{1}{d_{k}} \sum_{l \in \mathcal{N}_{k}}\left(z_{t}^{l}-z_{t}^{k}\right) \Omega_{t}^{k}+\left(1-a_{t}+a_{t} \Omega_{t}^{k}\right) z_{t}^{k}\right] .
\end{aligned}
$$

Subsequently, we obtain

$$
\begin{aligned}
z_{t+1}^{k} & =\frac{1}{\rho_{k}}\left[a_{t} \frac{\Omega_{t}^{k}}{d_{k}} \sum_{l \in \mathcal{N}_{k}}\left(z_{t}^{l}-z_{t}^{k}\right)+\left(1-a_{t}+a_{t} \Omega_{t}^{k}\right) z_{t}^{k}\right] \\
& =\frac{1}{\rho_{k}}\left[a_{t} \frac{\Omega_{t}^{k}}{d_{k}} \mathbf{L}_{k} z_{t}+\left(1-a_{t}+a_{t} \Omega_{t}^{k}\right) z_{t}^{k}\right],
\end{aligned}
$$

where $\mathbf{L}_{k}$ is the $k$ th row of $\mathbf{L}$ and

$$
\rho_{k}=\left|a_{t} \frac{1}{d_{k}} \Omega_{t}^{k} \mathbf{L}_{k} z_{t}+\left(1-a_{t}+a_{t} \Omega_{t}^{k}\right) z_{t}^{k}\right| .
$$

(If $\rho_{k}=0$, then agent $k$ is allowed to take any position on the circle [12].)

We define the following additional matrices: $\mathbf{D}_{\rho}=$ $\operatorname{diag}\left(\rho_{1}^{-1}, \ldots, \rho_{N}^{-1}\right)$ and $\mathbf{D}_{\Omega}=\operatorname{diag}\left(\Omega_{t}^{1}, \ldots, \Omega_{t}^{N}\right)$. Recall $\mathbf{D}_{d}=\operatorname{diag}\left(d_{1}^{-1}, \ldots, d_{N}^{-1}\right)$, which for all-to-all communication becomes $\mathbf{D}_{d}=\frac{1}{N-1} I_{N}$. We have

$$
z_{t+1}=\mathbf{D}_{\rho}\left[a_{t} \mathbf{D}_{d} \mathbf{D}_{\Omega} \mathbf{L} z_{t}+\left(\left(1-a_{t}\right) I_{N}+a_{t} \mathbf{D}_{\Omega}\right) z_{t}\right]
$$

Substituting (19) into (17) gives

$$
\begin{aligned}
P_{t+1}= & {\left[a_{t} \mathbf{D}_{d} \mathbf{D}_{\Omega} \mathbf{L} z_{t}+\left(\left(1-a_{t}\right) I_{N}+a_{t} \mathbf{D}_{\Omega}\right) z_{t}\right]^{T} } \\
& \mathbf{D}_{\rho} \mathbf{L D}_{\rho}\left[a_{t} \mathbf{D}_{d} \mathbf{D}_{\Omega} \mathbf{L} z_{t}+\left(\left(1-a_{t}\right) I_{N}+a_{t} \mathbf{D}_{\Omega}\right) z_{t}\right]
\end{aligned}
$$

Let $\hat{\mathbf{L}}=\mathbf{D}_{d} \mathbf{L}$ and $\mathbf{L}^{\prime}=\mathbf{D}_{\rho} \mathbf{L} \mathbf{D}_{\rho}$. Since $\mathbf{L}, \mathbf{D}_{d}$, and $\mathbf{D}_{\rho}$ are symmetric positive semi-definite, $\hat{\mathbf{L}}$ and $\mathbf{L}^{\prime}$ are symmetric positive semi-definite. Consider the following equalities:

$$
\begin{aligned}
{\left[a_{t} \mathbf{D}_{d} \mathbf{D}_{\Omega} \hat{\mathbf{L}} z_{t}\right]^{T} } & =a_{t} z_{t}^{T} \hat{\mathbf{L}} \mathbf{D}_{\Omega} \\
{\left[\left(\left(1-a_{t}\right) I_{N}+a_{t} \mathbf{D}_{\Omega}\right) z_{t}\right]^{T} } & =\left(1-a_{t}\right) z_{t}^{T}+a_{t} z_{t}^{T} \mathbf{D}_{\Omega} .
\end{aligned}
$$


The total potential is

$$
\begin{aligned}
P_{t+1}= & \left(1-a_{t}\right)^{2} z_{t}^{T} \mathbf{L}^{\prime} z_{t} \\
& +a_{t}\left(1-a_{t}\right)\left(z_{t}^{T} \hat{\mathbf{L}} \mathbf{D}_{\Omega} \mathbf{L}^{\prime} z_{t}+z_{t}^{T} \mathbf{L}^{\prime} \mathbf{D}_{\Omega} \hat{\mathbf{L}} z_{t}\right. \\
& \left.+z_{t}^{T} \mathbf{L}^{\prime} \mathbf{D}_{\Omega} z_{t}+z_{t}^{T} \mathbf{D}_{\Omega} \mathbf{L}^{\prime} z_{t}\right) \\
& +a_{t}^{2}\left(z_{t}^{T} \hat{\mathbf{L}} \mathbf{D}_{\Omega} \mathbf{L}^{\prime} \mathbf{D}_{\Omega} \hat{\mathbf{L}} z_{t}+z_{t}^{T} \hat{\mathbf{L}} \mathbf{D}_{\Omega} \mathbf{L}^{\prime} \mathbf{D}_{\Omega} z_{t}\right. \\
& \left.+z_{t}^{T} \mathbf{D}_{\Omega} \mathbf{L}^{\prime} \mathbf{D}_{\Omega} \hat{\mathbf{L}} z_{t}+z_{t}^{T} \mathbf{D}_{\Omega} \mathbf{L}^{\prime} \mathbf{D}_{\Omega} z_{t}\right)
\end{aligned}
$$

Taking the expectation of both sides of (20) builds the Lyapunov function expression (18). Analysis of (18) is ongoing, but suggest the following proposition.

Proposition: Under assumptions $\left(\mathrm{A} 1^{\prime}\right)-\left(\mathrm{A} 3^{\prime}\right)$, algorithm (16) achieves weak consensus on the $N$-torus, i.e.,

$$
\lim _{t \rightarrow \infty} E\left|z_{t}^{k}-z_{t}^{l}\right|^{2}=0 \forall k, l \in \mathcal{N} \text {. }
$$

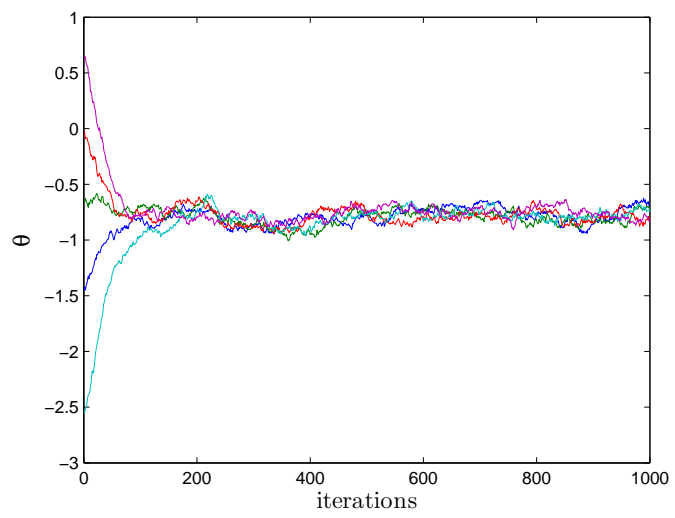

Fig. 7. Five agents reach weak consensus on the torus with noise standard deviation $\sigma=10$.

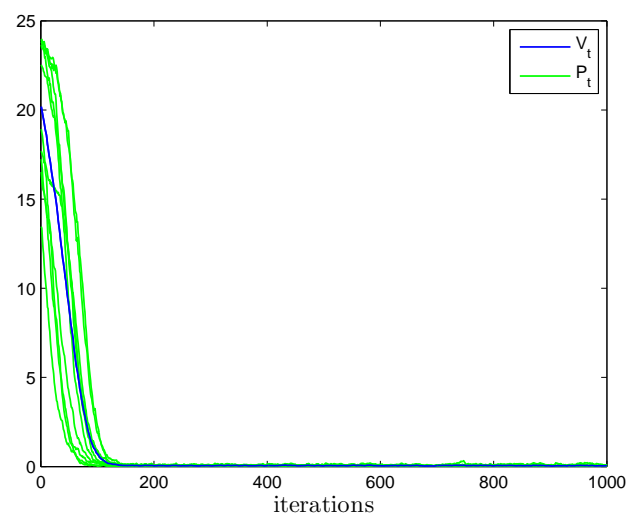

Fig. 8. The potential of five agents for ten trials using $\sigma=10$ and the stochastic Lyapunov function.

Figure 7 shows the weak convergence of algorithm (16) for five agents with all-to-all communication where the measurement noise has normal distribution and is randomly selected between $[-\pi / 2, \pi / 2]$, with standard deviation $\sigma=$ 10 and zero mean. Figure 8 illustrates the potential $P_{t}$ for ten trials and shows that the resulting stochastic Lyapunov function $V_{t}$ decays to zero.

\section{CONCLUSIONS}

We are considering the problem of cooperative control of unmanned vehicles in an uncertain environment when each vehicle can obtain only noisy measurements of the other vehicles. We aim to prove convergence to the desired formation and robustness to measurement errors using a stochastic algorithm. This paper studies the problem of consensus on the $N$-torus for all-to-all interactions corrupted by noise. A stochastic approximation algorithm is applied and weak consensus of agents is established. In ongoing research we are extending the results to limited communication.

\section{REFERENCES}

[1] Juan Acebrn, L. Bonilla, Conrad Prez Vicente, Flix Ritort, and Renato Spigler. The Kuramoto model: A simple paradigm for synchronization phenomena. Review of Modern Physics, 77(1):137-185, January 2005.

[2] Moayin Chen, Yun Shang, Yong Zou, and Jurgen Kurths. Synchronization in the Kuramoto model: A dynamical gradient network approach. Physical Review E, 77(2):027101-1-4, February 2008.

[3] Jorge Cortes. Global and robust formation-shape stabilization of relative sensing networks. Automatica, 45:2754-2762, 2009.

[4] Miniyi Huang and Jonathan H. Manton. Coordination and consensus of networked agents with noisy measurements: Stochastic algorithms and asymptotic behavior. SIAM Journal on Control and Optimization, 48(1):134-161, 2009.

[5] Miniyi Huang and Jonathan H. Manton. Stochastic consensus seeking with noisy and directed inter-agent communication: Fixed and randomly varying topologies. IEEE Transactions on Automatic Control, 55(1):235-241, January 2010.

[6] Ali Jadbabaie, J. Lin, and A. Steven Morse. Coordination of groups of mobile autonomous agents using nearest neighbor rules. IEEE Transactions on Automatic Control, 48(6):988-1001, June 2003.

[7] Luc Moreau. Stability of multi-agent systems with time-dependent communication links. IEEE Transactions on Automatic Control, 50(2):169-182, February 2005.

[8] Reza Olfati-Saber, Alex J. Fax, and Richard M. Murray. Consensus and cooperation in networked multi-agent systems. In Proceedings of the IEEE, volume 95, pages 215-233, January 2007.

[9] Wei Ren, Randal W. Beard, and Ella M. Atkins. A survey of consensus problems in multi-agent coordination. In Proceedings of the 2005 American Control Conference, pages 1859-1864, Portland, OR, June 2005.

[10] Alain Sarlette, Silvere Bonnabel, and Rodolphe Sepulchre. Corrdinated motion design on Lie groups. IEEE Transactions on Automatic Control, 55(5):1047-1058, 2010.

[11] Alain Sarlette, Rodolphe Sepulchre, and Naomi Ehrich Leonard. Discrete-time synchronization on the $N$-torus. In Proceeding of the 17th International Symposium on Mathematical Theory of Networks and Systems, pages 2408-2411, Kyoto, Japan, 2006.

[12] Luca Scardovi, Alain Sarlette, and Rodolphe Sepulchre. Synchronization and balancing on the $N$-torus. Systems and Control Letters, 56(5):335-341, 2007.

[13] R. Sepulchre, D. A. Paley, and N. E. Leonard. Stabilization of planar collective motion: All-to-all communication. IEEE Transactions on Automatic Control, 52(5):811-824, 2007.

[14] M. K. Stephen Yeung and Steven H. Strogatz. Time delay in the Kuramoto model of coupled oscillators. Physical Review Letters, 82(3):648-651, January 1999.

[15] George F. Young, Luca Scardovi, and Naomi E. Leonard. Robustness of noisy consensus dynamics with directed communication. In Proceedings of the 2010 American Control Conference, pages 6312-6317, Baltimore, MD, June 2010. 